\title{
The black silicon method: a universal method for determining the parameter setting of a fluorine-based reactive ion etcher in deep silicon trench etching with profile control
}

\author{
Henri Jansen, Meint de Boer, Rob Legtenberg and \\ Miko Elwenspoek
}

MESA Research Institute, University of Twente, PO Box 217, 7500 AE Enschede, The Netherlands

Received 14 December 1994, accepted for publication 4 January 1995

\begin{abstract}
Very deep trenches (up to $200 \mu \mathrm{m}$ ) with high aspect ratios (up to 10) in silicon and polymers are etched using a fluorine-based plasma $\left(\mathrm{SF}_{6} / \mathrm{O}_{2} / \mathrm{CHF}_{3}\right)$. Isotropic, positively and negatively (i.e. reverse) tapered as well as. fully vertical walls with smooth surfaces are achieved by controlling the plasma chemistry. A convenient way to find the processing conditions needed for a vertical wall is described: the black silicon method. This new procedure is checked for three different reactive ion etchers (RIE), two parallel-plate reactors and a hexode. The influence of the RF power, pressure and gas mixture on the profile will be shown. Scanning electron microscope (SEM) photos are included to demonstrate the black silicon method, the influence of the gases on the profile, and the use of this method in fabricating microelectromechanical systems (MEMS).
\end{abstract}

\section{Introduction}

Silicon profile control is an important topic in microtechnology. Especially, vertical walls are needed to get high feature densities. Most commonly, wet chemical etchants are used to create anisotropic profiles, because they are cheap and easy to use. However, the etched profile is controlled by the crystal orientation, so there is minor freedom in etching different tapered profiles. Dry plasma etching is becoming a standard tool in microtechnology. Although the basic investments are much higher in dry etching, it is possible to etch controllable profiles without using the crystal orientation. Plasma etching can be divided into three main groups; the physical ion beam etching (IBE), the synergetic reactive ion etching (RIE), and the chemical plasma etching (PE). Generally, IBE shows only positively tapered profiles, low etch rates and low selectivity, whereas PE gives rise to isotropic profiles, high etch rates and high selectivity. In RIE it is possible to provide the plasma with a chemical etchant for the etching of the substrate, a passivator for blocking the etching at the sidewalls of a trench, and an ion surce for the local removal of the passivation layer at the bottom of the etching trenches. When these processes are controlled in the correct manner, it is possible to create all kinds of trenches with excellent profile control, high etch rates and selectivity. RIE processes which use the deposition of a passivating film to block spontaneous etching are known as ion-inhibitor RIE. To etch polymers it is enough to prevent spontaneous etching by lowering the temperature well below the glass-temperature. In these cases the etching is made possible because of ion bombardment. This is a typical reactive ion beam etching (RIRE) process, but can also be fulfilled at higher pressures with a RIE apparatus. RIE processes which use only the incoming ions to achieve directionality are called: ion-induced RIE. To increase the etch rate, standard RIE is modified to create a higher-density plasma [1-6], but these etchers are expensive and therefore less attractive.

Normally, halogen-based plasmas are used for the chemical etching of silicon [1-14], because of their high etch rates. Except for the fluorine-based plasmas, these gases are particularly hazardous (e.g. chlorine, bromine and chlorinated compounds) and special precautions are recommended. The passivation layer can be grown: (i) from carbonhalogen polymer precursors which are let in to the plasma [8-10]; (ii) by redeposition of metalhalogen mask material [11]; (iii) by inserting gases which act as an oxidant forming siliconoxyhalogens [1,12-14]; or (iv) by freezing the normally volatile siliconhalogen reaction products of the silicon with the radicals $[3,4]$. The 


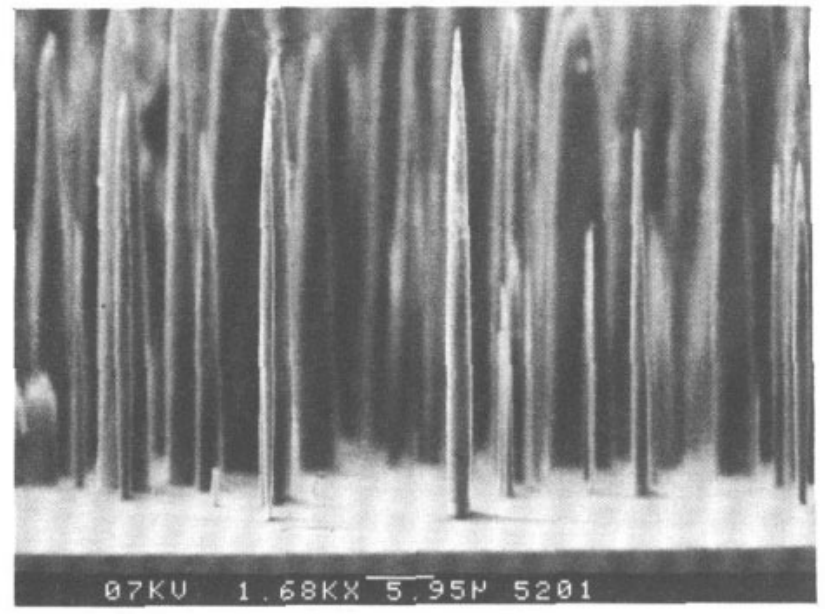

Figure 1. The formation of grass.

deposition of a polymer film has the disadvantage that this film is thermally less stable than a growing inorganic siliconoxyhalogen film and the freezing of reaction products uses expensive (cryogenic) coolers. The redeposition of mask material is not acceptable because areas which should stay clean are also contaminated. Because the passivating film is very thin, the incoming ions should not be highly energetic, so the selectivity will be very high and the substrate damage will be low. Also, because of the low energy of the ions, trenching and faceting are not found, and it is very easy to change the direction of the impinging ions, thus changing the etched profile. A major problem during etching silicon vertically, is the forming of 'grass' on the silicon surface, because of all kinds of micromasks deposited or grown on the silicon [14] (figure 1).

To etch polymers, commonly low pressure oxygen plasmas are used. However, such a plasma creates a high DC bias voltage which is responsible for substrate- and mask-damage. During etching a polymer in low pressure RIE identical grass problems are observed as in the case of etching silicon.

In this study, the low-toxic and easy-to-handle $\mathrm{SF}_{6} / \mathrm{O}_{2} / \mathrm{CHF}_{3}$ gases are chosen for the reactive ion etching of silicon and polymers [14]. A short description of the etch mechanism of silicon and polymers in such a plasma is given and a new method to find the fully vertical profile regime is introduced which uses the forming of grass: the black silicon method. When this regime is found, $\mathrm{CHF}_{3}$ is added to prevent the forming of grass. It is shown that this method is a practical tool for finding the parameter setting of an arbitrary RIE. The influence of the RF power, pressure and gas composition on, and its relation to, the profile is given in a diagram. With the help of this diagram it is easy to direct the chemistry for the desired profile. The aim of this work is the fabrication of high-aspect-ratio (depth/width) features for use in microelectromechanical systems (MEMS). As an example, a MEM comb driven $x y$ stage is given.

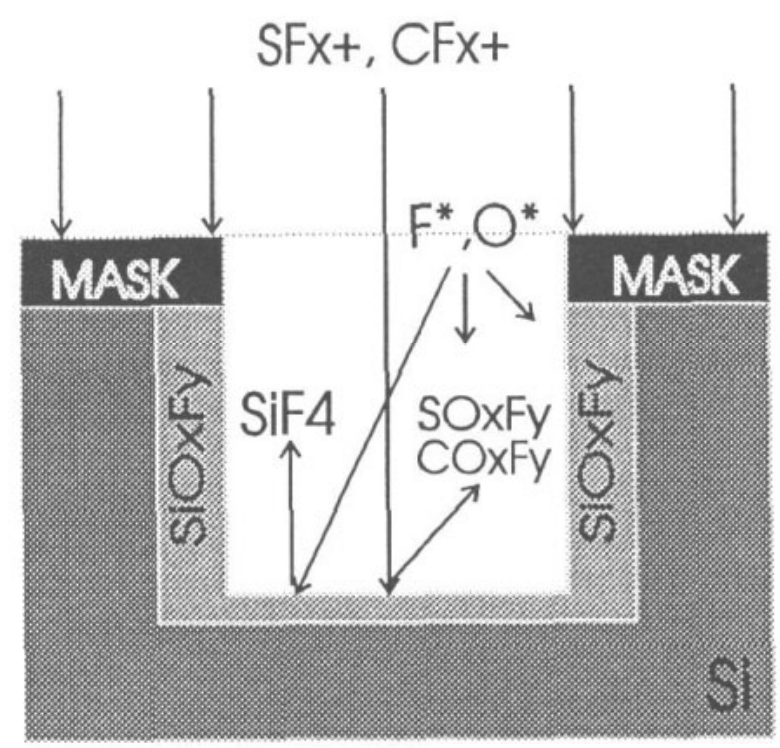

Figure 2. The $\mathrm{SF}_{6} / \mathrm{O}_{2} / \mathrm{CHF}_{3}$ chemistry.

\section{The synergetic mechanism of $\mathrm{SF}_{6} / \mathrm{O}_{2} / \mathrm{CHF}_{3}$ plasmas}

In an $\mathrm{SF}_{6} / \mathrm{O}_{2} / \mathrm{CHF}_{3}$ plasma, each gas has a known specific function and influence, so the etched profile is easily controlled just by changing the flow rate of one of these gases [14]. In such a plasma $\mathrm{SF}_{6}$ produces the $\mathrm{F}^{*}$ radicals for the chemical etching of the silicon forming the volatile $\mathrm{SiF}_{4} ; \mathrm{O}_{2}$ creates the $\mathrm{O}^{*}$ radicals to passivate the silicon surface with $\mathrm{SiO}_{x} \mathrm{~F}_{y}$; and $\mathrm{CHF}_{3}$ is the source for the $\mathrm{CF}_{x}^{+}$ ions which etch the $\mathrm{SiO}_{x} \mathrm{~F}_{y}$ layer in one direction forming the volatile $\mathrm{CO}_{x} \mathrm{~F}_{y}$ (see figure 2). Of course, $\mathrm{SF}_{x}^{+}$ions are also able to remove the oxyfluoride by way of the volatile $\mathrm{SO}_{x} \mathrm{~F}_{y}$ gases, but the $\mathrm{SF}_{6}$ flow is fixed on the $\mathrm{O}_{2}$ flow to ensure a vertical wall. Thus, the $\mathrm{CHF}_{3}$ gas is a nearly independent source of oxyfluoride-etching ions.

A more or less contrary mechanism can also explain the directional etching. In this mechanism the $\mathrm{CF}_{x}$ species are passivating the silicon surfaces which are etched by way of imparting $\mathrm{O}^{+}$ions. However, this mechanism is less likely in the pressure regime used in our study as will be clarified further on.

The $\mathrm{SF}_{6} / \mathrm{O}_{2} / \mathrm{CHF}_{3}$ chemistry is able to etch highly controllable profiles in silicon at very low ion energies (20$90 \mathrm{eV}$ ) and high etch rates (up to $3 \mu \mathrm{m} \mathrm{min}{ }^{-1}$ ). The low ion energy prevents substrate damage (electronics), mask erosion (the selectivity to metal masks is practically infinite) and makes it easy to change the profile of the trench. The ion energy is ruled by the potential which is developed between the plasma and the powered electrode, the DC selfbias. Gases like $\mathrm{O}_{2}$ and $\mathrm{CHF}_{3}$ create high bias voltages whereas $\mathrm{SF}_{6}$ gives rise to very low voltage [14]. Thus, when the oxygen flow is increased the DC self-bias also increases and ions will gain more energy before colliding with the substrate surface. The DC self-bias decreases when the power decreases or the pressure increases.

In etching silicon with the $\mathrm{SF}_{6} / \mathrm{O}_{2} / \mathrm{CHF}_{3}$ mixture there is a constant competition between the etching fluorine radicals and the passivating oxygen radicals. The etching 


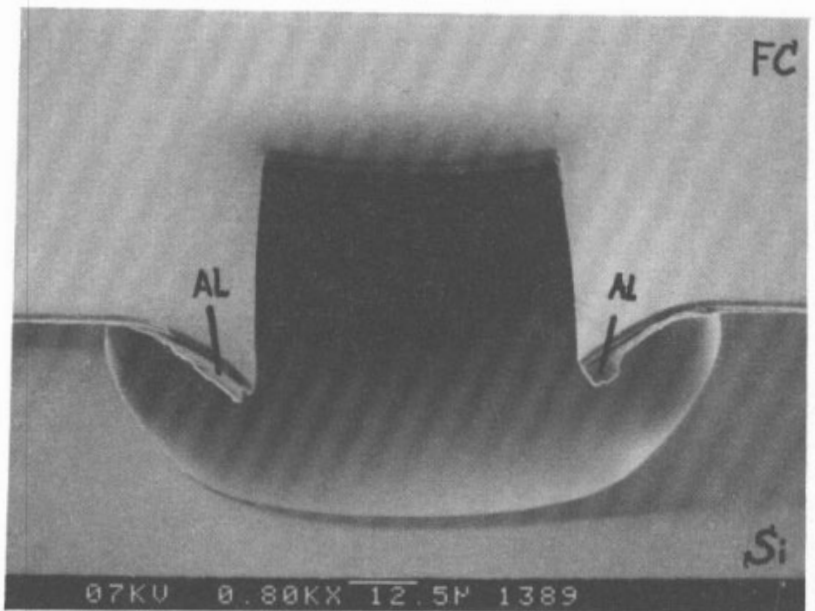

Figure 3. An isotropic profile.

is increased directionally by way of the $\mathrm{CF}_{x}^{+}$ions. When the $\mathrm{SF}_{6}$ content is increased, the formation of the blocking layer is less pronounced and therefore the profile will be more isotropic (i.e. PE-like, figure 3). Increasing the oxygen content will decrease the chemical etching and the etch mechanism will become less isotropic. At higher oxygen concentrations the etching will become physical, which results in positively tapered profiles (i.e. IBE-like, figure 4). Increasing the $\mathrm{CHF}_{3}$ content will increase the removal of the blocking layer, thus making the profile less positively tapered. Moreover, the ions are charged positively, whereas the substrate is negatively biased and because of this mechanism it is possible to create negatively (i.e. reverse) tapered profiles due to ion bowing (figure 5). At higher $\mathrm{CHF}_{3}$ concentrations $\mathrm{CF}_{x}$ specimens will scavenge the oxygen radicals, thus preventing the blocking layer from forming, which results in a more isotropic profile. When the power, pressure, and flows are in the correct balance, vertical wall profiles result (figure 6). Note that in this figure two less attractive effects are observed: (i) when the line spacing is wider, the trenches are deeper. This effect is known as 'RIE-lag'. (ii) The bigger areas have negatively tapered wall profiles because of ion bowing. Both effects will be described in a follow-up of this paper [15]. A higher pressure or lower power results in a more positively tapered profile, because the energy of the impinging ions is lower (DC self-bias). In these cases, off-normal ions are more likely to reflect from the side wall without etching it. When the etching is performed in the isotropic or negatively tapered regime, thus at low oxygen, high $\mathrm{CHF}_{3}$ flow, low pressure, or high RF power, micromasks such as native oxide, dust, or redeposited mask material will be constantly underetched and etched surfaces stay smooth, preventing the forming of grass (figure 7).

When the present process is used in etching silicon structures, the silicon loading of the substrate should be sufficiently high, e.g. at least $10 \%$. If the substrate has a lower silicon loading, additional silicon may be added to the etching chamber in any form. Even more important, the wafer temperature must be stable and low enough to ensure controllable profiles. Most times vacuum grease together with a cooling system at $20^{\circ}$ will do very well.

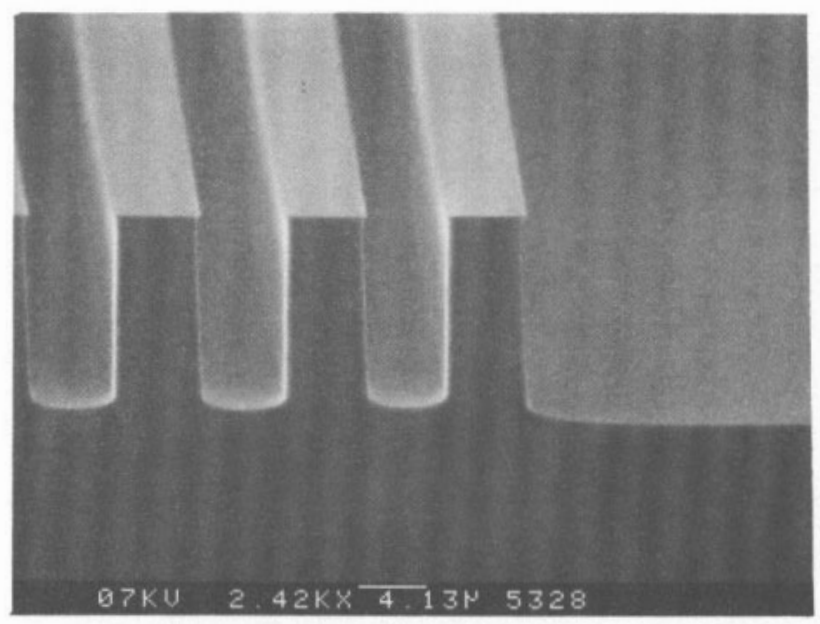

Figure 4. Positive tapered profile.

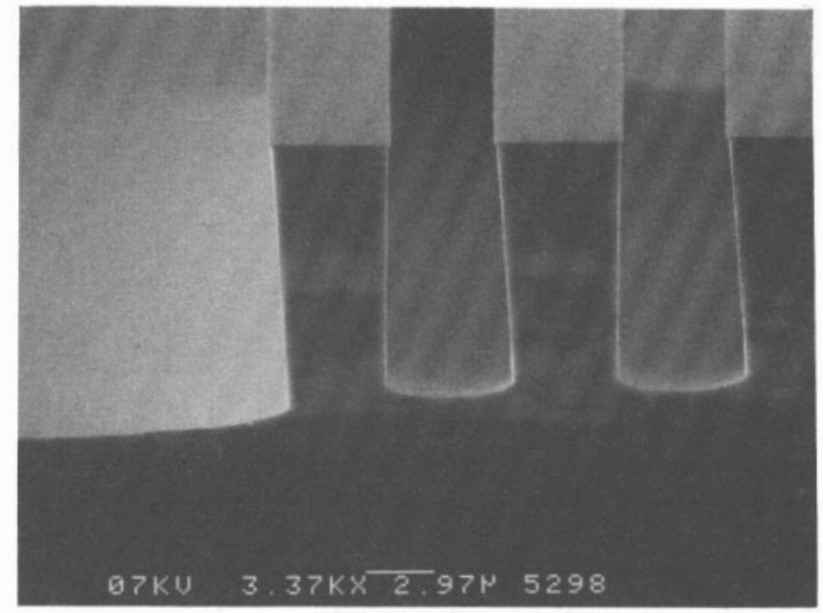

Figure 5. A negative tapered profile.

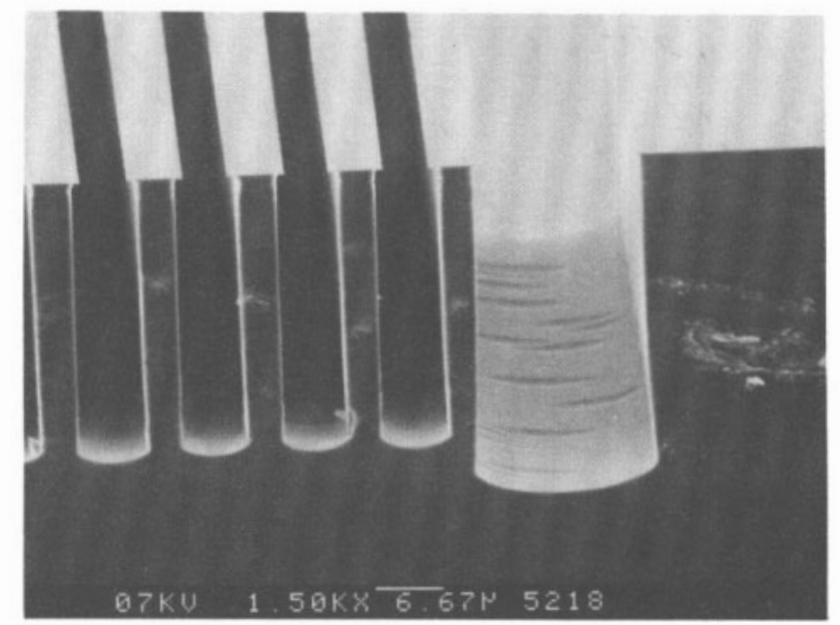

Figure 6. A vertical wall profile.

It is also possible to etch polymers, instead of silicon with high etch rates and aspect ratios with the same $\mathrm{SF}_{6} / \mathrm{O}_{2} / \mathrm{CHF}_{3}$ plasma, but in these cases the specific function of each gas is different. Now, $\mathrm{O}_{2}$ creates the $\mathrm{O}^{*}$ radicals to etch the polymer chemically, but this etching is highly temperature dependent. At temperatures near or above the polymer glass transition temperature, the etch 


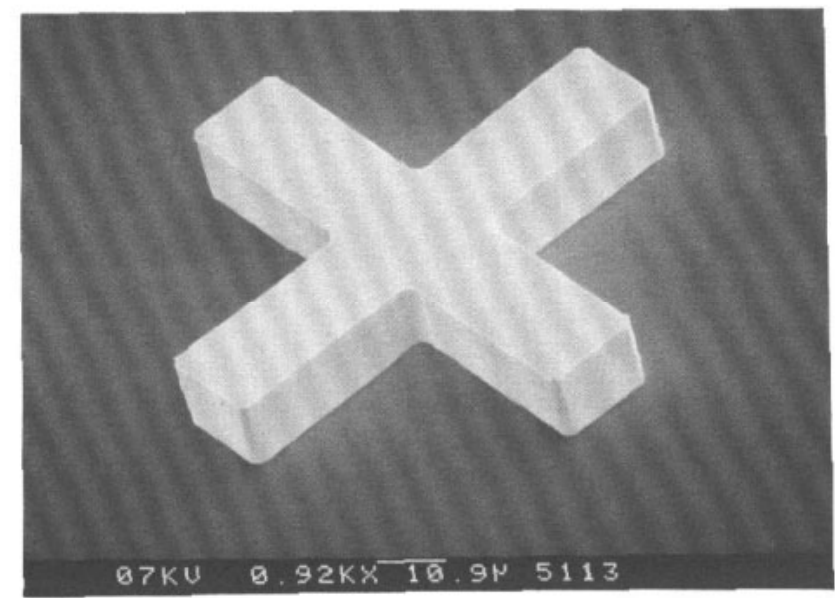

Figure 7. An etched structure.

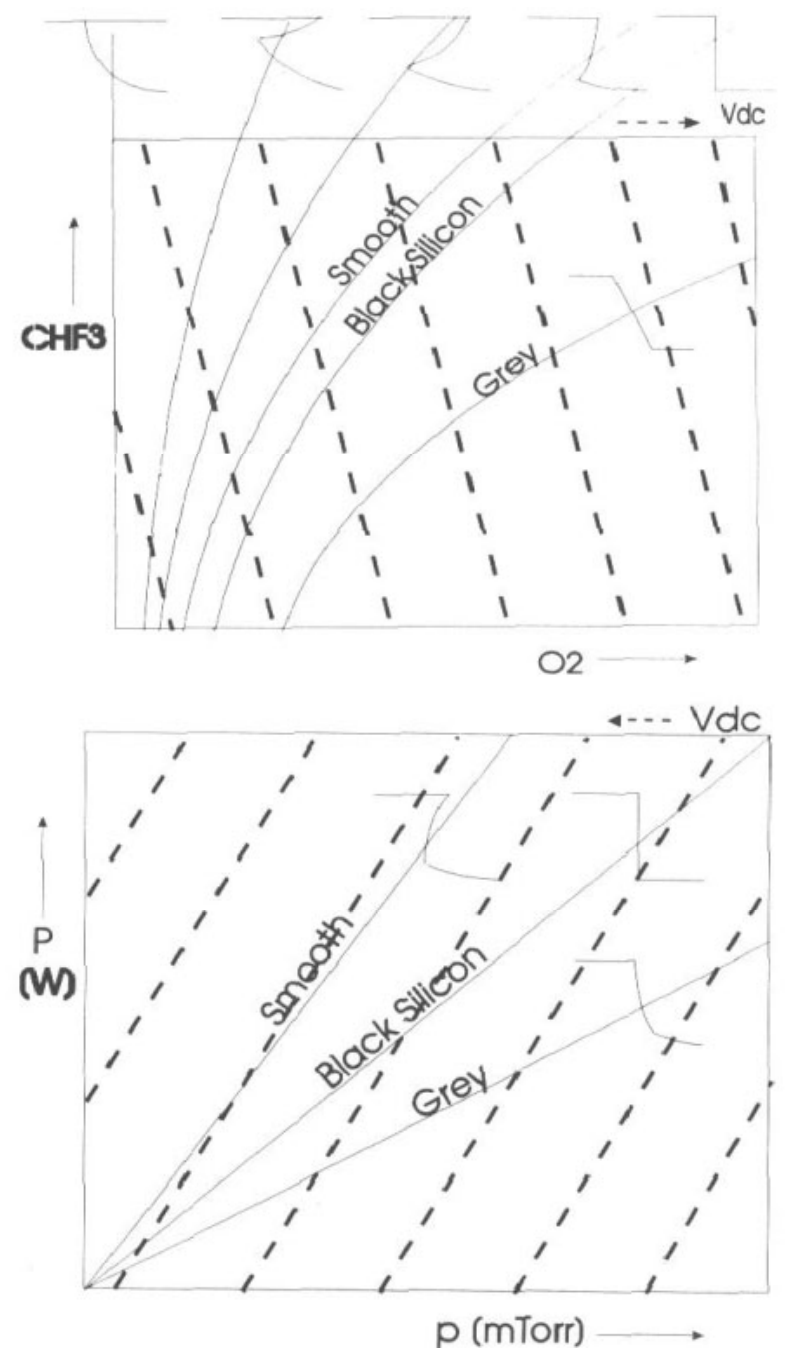

Figure 8. The influence of power, pressure and flow on the profile.

profile is purely isotropic and the etch rate can be as high as 5 microns per minute. This process is normally fulfilled in so-called plasma ashing systems to strip resist after mask duplication. In order to create anisotropic profiles, it is necessary to block this thermo-chemical etching, so the

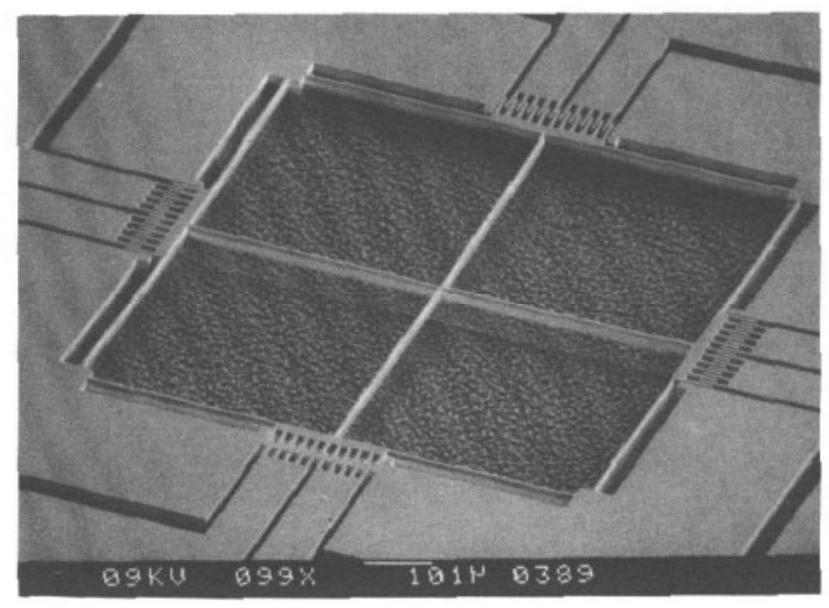

Figure 9. A micromachined $x y$ stage etched with the help of the black silicon method in an $\mathrm{SF}_{6} / \mathrm{O}_{2} / \mathrm{CHF}_{3}$ plasma.

substrate is cooled at room temperature and at the same time $\mathrm{O}^{+}$ions are used to etch the trench bottom. For this reason RIE is used to direct the ions from the plasma glow region towards the substrate. Because of the highly anisotropic etch, grass will appear just as in the case of the RIE of silicon. To prevent this formation of grass, again $\mathrm{CHF}_{3}$ is added resulting in nearly vertical and smooth surfaces. To lower the high DC bias voltage (thus increasing the mask selectivity) which is created during $\mathrm{O}_{2} / \mathrm{CHF}_{3}$ reactive ion etching $\mathrm{SF}_{6}$ and dummy-silicon are added giving very low bias voltages (down to $10 \mathrm{eV}$ ). The addition of $\mathrm{CHF}_{3}$ as well as $\mathrm{SF}_{6}$ is not influencing the polymer etch rate (up to $2 \mu \mathrm{m} \mathrm{min}^{-1}$ ) more than $10 \%$.

\section{The black silicon method}

Of course, it is possible to continue this paper by giving the recipe for the RIE giving a vertical wall profile and show the influence of the various parameters on this profile such as power, pressure and gas flow. However, this information cannot be used in other laboratories, especially when they have a different reactor. This problem has its origin in the difference of the configuration of the etcher (plan-parallel diode, triode, hexode, etc.) but also in the reactor geometry, the flow handling (turbo-, diffusion pump, etc.) and many others. For this reason a stronger tool is used to find the vertical profile regime. This method uses the fact that the silicon is turned black when the vertical wall recipe is found. From now on, this method will be called the 'black silicon method'. Another method using response surface methodology has previously been described by the authors [14]; it includes information over the equipment and the actual recipe. Before the black silicon method is formulated, the reason for this effect will be explained and a way to get rid of this blackening will be described.

\subsection{The origin of black silicon}

As stated in the above there is a constant competition between the fluorine radicals that etch and the oxygen radicals that passivate the silicon. At a certain oxygen 
Table 1. The formulation of the black silicon method.

1. Place a piece of silicon in the reactor and adjust the preferred power and pressure for an $\mathrm{SF}_{6} / \mathrm{O}_{2}$ plasma. Etch ca. $1 \mu \mathrm{m}$ of silicon, open the process chamber, and look if the silicon is black. If not, do the same again but increase the oxygen flow. Proceed with this sequence until the wafer is black. Increasing the oxygen too much will still give rise to black-, or better grey-, silicon since there exists a positive tapered profile without any underetching. Alternatively, it is possible to sense the black siticon with the help of a laser/photodetector set-up. Note, be sure that the thermal contact of the sample with the e.g. water cooled powered electrode is properly controlled i.e. use vacuum grease, mechanical clamping, helium backside cooling, and so forth.

2. After the black silicon regime is found, add some $\mathrm{CHF}_{3}$ to the mixture and increase this flow until the wafer is clean again. Too much $\mathrm{CHF}_{3}$ will make the profiles isotropic (and smooth) because the $\mathrm{CF}_{x}$ species are scavenging the oxygen radicals needed for the blocking layer.

3. Now a wafer with the mask pattern of interest is inserted in the reactor and the etched profile is checked. Increasing the $\mathrm{SF}_{6}$ content will create a isotropic profile (figure 3 ). Adding too much oxygen will make the profile positively tapered (figure 4) and extra $\mathrm{CHF}_{3}$ will make it more negatively tapered (figure 5). Adding at the same $\mathrm{O}_{2}$ and $\mathrm{CHF}_{3}$ with the correct balance will create very smooth and nearly vertical walls (figures 6,7 ) (figure 6 ). Increasing the pressure or decreasing the power will make the profile more positively tapered. In figure 8 the influence of $\mathrm{O}_{2} / \mathrm{CHF}_{3}$ flow and the pressure/power on the profile is given. Increasing at the same time the $\mathrm{O}_{2}$ and $\mathrm{CHF}_{3}$ flow, pressure and $\mathrm{CHF}_{3}$ flow, power and $\mathrm{O}_{2}$ flow, or power will hardly change the profile. However, such an increase will increase the DC self-bias and a higher $\mathrm{DC}$ self-bias will give the off-normal ions enough energy to etch the side walls, thus changing the profile a little. Structure heights of $100 \mu \mathrm{m}$ with an undercut of less than $1 \mu \mathrm{m}$ are achieved.

content there is such a balance between the etching and the passivation that a nearly vertical wall results. At the same moment native oxide, dust, etc. will act as micromasks and, because of the directional etching, spikes will appear. These spikes consist of a silicon body with a thin passivating silicon oxyfiuoride skin. They will become higher in time and, depending on the etch rate, they will exceed the wavelength of incoming light after some time. This light will be 'caught' in the areas between the spikes and cannot leave the silicon surface any more. So, all the light is collected by the etching surface and it is turned black. In fact, this optical diffuser could be used for all kinds of applications where the refiection of light from the surroundings is not desired, e.g. laser applications or sunlight collectors. In figure 1, a SEM photo is shown from etched silicon under directional conditions. The spikes are $50 \mu \mathrm{m}$ in height and ' a few $\mu \mathrm{m}$ in width. The origin of micromasks is caused by native oxide, dust and so on which is already on the wafer before etching. But, it is also formed during the etching because silicon oxide particles coming from the plasma are adsorbing at the silicon surface or because of the oxidation of the silicon surface together with the angle-dependent ion etching of this oxide layer. Another source of particles during etching which will act as micromasks is the redepositioning of mask material due to imparting ions.

\subsection{Preventing black silicon}

Spikes formed because of dirty wafers before etching are easily controlled by giving the wafer a precleaning step. For instance, native oxide can be removed with the help of an HF dip and dust is less of a problem when using the lift-off technique in applying the mask layer, instead of the normally used chemical etching of the mask material with the help of a resist pattern. However, the micromasks which originate during etching must be controlled in a different way. First of all, the redeposition of mask material can be suppressed when the ion energy is low or when the right materials are chosen, but this subject will be treated elsewhere [15]. The silicon oxide particles are less of a problem when the selectivity between the silicon and the silicon oxide is minimized, but this only occurs when the incoming ions are highly energetic and at these moments the process is not favourable any more because of substrate damage and the just mentioned mask erosion. As already stated, it is possible to forbid spikes from forming by constantly underetching the micromasks isotropically or etching the features with a slightly negative undercut. The isotropic solution only makes sense when it is used as a post-etch, because otherwise the feature density is limited. On the other hand, negative underetching is an excellent way to control the smoothness of the substrate surface barely limiting the feature size density. In this study the addition of $\mathrm{CHF}_{3}$ to an $\mathrm{SF}_{6} / \mathrm{O}_{2}$ plasma is described and its ability to prevent grass. Yet another approach to attack the grass problem is the application of different masks, but this will be published elsewhere [15].

\subsection{The black silicon method}

In table 1 , an easy way to find the vertical wall regime is described with the help of the information already given. A more or less general tool is reached in which the recipe for any RIE system can be found just by fulfilling the sequence written down. As can be concluded from point 3 of this sequence, purely vertical walls can be achieved for any pressure, power, $\mathrm{O}_{2}, \mathrm{CHF}_{3}$, or $\mathrm{SF}_{6}$ flow. This is an important conclusion because now we are able to create any DC self-bias we want without changing the profile. For instance, it is possible to develop very low bias voltages $(<20 \mathrm{eV})$ at higher pressures giving very high mask selectivity, maintaining the profile (the sputter threshold below which no sputtering takes place usually is in the $20-40 \mathrm{eV}$ range). In such cases the etched silicon bottom and the side walls are nearly perfect as shown in figure 7. It is also observed in figure 8 that a vertical wall profile is found for zero $\mathrm{CHF}_{3}$ flow. This means that the passivation with silicon oxyfluorides at the side walls is more likely than the passivation with a fluorocarbon layer, 
although it is still possible that a different pressure, loading, etc., the fluorocarbon layer is more pronounced. Also, the observation that increasing the oxygen flow gives rise to a more positively tapered profile is a strong indication that silicon oxyfluoride is the side wall passivator. Auger analysis showed that indeed the side walls are covered with silicon oxide; there is no carbonic species found [14].

The black silicon method is tested for three different RIE systems. Most experiments are performed with a plan-parallel plate reactor 'Plasmafab 340 ' from the STS Company [14] and a second plan-parallel plate single-wafer reactor 'Plasmafab 500' showed identical results. A third system, the hexode 'AME-8100' from Applied Materials, is used for the batch fabrication of silicon wafers and is also able to achieve vertical profiles. However, the etch rates are approximately one order of magnitude lower than for the single-wafer etchers and for this reason less powerful. This is because the wafers are exposed for much longer to the aggressive plasma chemistry, giving rise to surface roughening when etching very deep trenches in silicon. The etch rate can be increased by decreasing the reactor loading. However, decreasing the exposed silicon surface area too much (typically smaller than $10 \%$ 'open' silicon surface on the substrate wafer) will change the plasma chemistry (thus changing the profile beyond a point where adjustment of the other variables such as pressure, oxygen flow, etc., can no longer prevent the underetching of the mask. Nevertheless, it is possible to insert some extra silicon in the reactor to ensure a certain minimal loading. This subject will be treated more extensively elsewhere [15].

Although the black silicon method was originally developed for silicon trench etching, it is found that this method works for polymer trench etching as well. Although the appearance of a polymer surface after anisotropic etching is not black but rather diffuse the mechanism is the same. For this reason, a more general name for this method is chosen: the black substrate method.

\section{Applications and conclusions}

Wafers that are purposely not cleaned or even oxidized in an oxygen plasma in the black silicon regime can be used as an optical diffuser for e.g. laser applications. It is possible to create spikes at well defined locations in order to form a tip for use in AFM applications. In our study we are mainly interested in the use of the black silicon method for MEMS applications. In figure 9, a micromachined $x y$ stage is shown. The structure is etched during one run with standard RIE. After the directional etching, the side walls are passivated using a low-pressure $\mathrm{CHF}_{3}$ plasma and the $x y$ stage is etched free with the help of an isotropic $\mathrm{SF}_{6}$ plasma. In the same run the structure is passivated with a fluorocarbon layer using a high-pressure $\mathrm{CHF}_{3}$ plasma [16].
Although the black silicon method is tested for $\mathrm{SF}_{6} / \mathrm{O}_{2} / \mathrm{CHF}_{3}$ plasma only, it will also work for other silicon etch gases, e.g. $\mathrm{CF}_{4}, \mathrm{NF}_{3}, \mathrm{SiF}_{4}, \mathrm{CF}_{3} \mathrm{Br}$, or $\mathrm{Cl}_{2}$. In fact, every plasma mixture which consists of a chemical etchant, passivator and an ion source can be used for the black silicon method, even when the substrate is not silicon at all but e.g. a polymer. All together it is shown that the black silicon method is a very strong tool for etching high structures with excellent profile control using an $\mathrm{SF}_{6} / \mathrm{O}_{2} / \mathrm{CHF}_{3}$ plasma.

\section{References}

[1] D'Emie C P D, Chan K K and Blum J 1992 Deep trench plasma etching of SC Si using $\mathrm{SF}_{6} / \mathrm{O}_{2}$ gas mixtures $J$. Vac. Sci. Technol. B 10 1105-10

[2] Yeam G-Y, Ono Y and Yamaguchi T 1992 Polysilicon etchback plasma process using $\mathrm{HBr}, \mathrm{Cl}_{2}$ and $\mathrm{SF}_{6}$ gas mixtures for deep-trench isolation J. Electron. Soc. 139 $575-9$

[3] Petri R, Francou J-M, Inard A and Henry D 1991 Temperature effects on a RIPE reactor Microelectron. Eng. 13 459-62

[4] Tada T and Kanayama T 1993 Fabrication of Si nanostructures with electron beam lithography using AlN as a dry-etch durable resist $J$. Vac. Sci. Technol. B 11 2229-32

[5] Pornot C, Mahi B, Petit B, Arnal Y and Pelletier J 1986 Anisotropic etching of silicon using an $\mathrm{SF}_{6} / \mathrm{Ar}$ microwave multipolar plasma J. Vac. Sci. Technol. B 4 1-5

[6] Perry A J and Boswell R W 1989 Fast anisotropic etching of $\mathrm{Si}$ in an ICP reactor Appl. Phys. Lett. 55 148-50

[7] Winters H, Coburn J W and Chuang T J 1983 Surface processes in plasma-assisted etching environments $J$. Vac. Sci. Technol. B 1 469-80

[8] Krings A M, Eden $\mathrm{K}$ and Beneking $\mathrm{H} 1987$ RIE etching of deep trenches in Si using $\mathrm{CBrF}_{3}$ and $\mathrm{SF}_{6}$ plasma Microelectron. Eng. 6 553-8

[9] Shaw J K, Zhang Z L and MacDonald N C 1994 SCREAM I: a single mask SC Si, RIE process for MEMS Sensors Actuators A 40 63-70

[10] Yunkin V A, Fischer D and Voges E 1994 Highly anisotropic selective RIE of deep trenches in Si Microelectron. Eng. 23 373-6

[11] Gobrecht J Anisotropy enhancement by sidewall protection using an Al-mask in etch rate $\mathrm{SF}_{6}$ based RIE processes

[12] Syau T, Baliga B J and Hamaker R W 1991 RIE of Si trenches using $\mathrm{SF}_{6} / \mathrm{O}_{2}$ gas mixtures $J$. Electrochem. Soc. 138 3076-81

[13] Zhang J M, Li J Z and Wolf E D 1983 RIE for submicron structures of refractory metal silicides and polycides $J$. Vac. Sci. Technol. B 1 1037-42

[14] Legtenberg $R$, Jansen $H$, de Boer $M$ and Elwenspoek $M$ 1995 Anisotropic RIE of $\mathrm{Si}$ using $\mathrm{SF}_{6} / \mathrm{O}_{2} / \mathrm{CHF}_{3}$ gas mixtures $J$. Electron. Soc.

[15] Jansen $H \mathrm{~V}$, de Boer $M$, Legtenberg $R$ and Elwenspoek $M$ 1995 The black silicon method: the influence of masks and loading on Si trench etching in RIE MNE (Davos, Sept 1994) 27 475-80

[16] Jansen H V, Gardeniers J G E, Elders J, Tilmans H A C and Elwenspoek M 1994 Applications of fluorocarbon polymers in micromechanics and micromachining Sensors Actuators A 41-42 136-40 